LBL-34072

UC-410

\title{
CAPTURE FROM PAIR PRODUCTION AS A BEAM LOSS MECHANISM FOR HEAVY IONS AT RHIC
}

\author{
B. FEINBERG, A. BELKACEM, R. BOSSINGHAM, and H. GOULD \\ ACCELERATOR and FUSION RESEARCH DIVISION \\ Lawrence Berkeley Laboratory \\ University of California \\ Berkeley, CA 94720 \\ and \\ W.E. MEYERHOF \\ Stanford University \\ Stanford, CA 94305
}

MAY 1993

This work was supported by the Director, Office of Energy Research, Office of Basic Energy Sciences, Materials Sciences Division, of the U.S. Department of Energy under Contract No. DE-AC03-76SF00098. 


\title{
Capture From Pair Production as a Beam Loss Mechanism for Heavy Ions at RHIC*
}

\author{
B. Feinberg, A. Belkacem, R. Bossingham, Harvey Gould \\ Lawrence Berkeley Laboratory \\ 1 Cyclotron Rd., Berkeley, CA 94720 USA \\ W.E. Meyerhof \\ Stanford University \\ Stanford, CA 94305 USA
}

\section{Abstract}

Electron capture from electron-positron pair production is predicted to be a major source of beam loss for the heaviest ions at RHIC. Achieving the highest luminosity thus requires an understanding of the capture process. We report the first observation and measurement of this process, in Bevalac experiments using $1 \mathrm{GeV} / \mathrm{u} \mathrm{U}^{92+}$ projectiles on $\mathrm{Au}$ targets. Capture from pair production is a process in which the very high electromagnetic field involved in the collision of two relativistic heavy ions polarizes the vacuum, resulting in the production of an electron-positron pair and the capture of the electron by one of the ions. There are many theoretical papers published on capture from pair production with very large discrepancies between predicted cross sections. The experimental results are compared to theory, and the implications of extrapolations to RHIC energies are presented.

\section{INTRODUCTION}

At relativistic energies, the capture of electrons by ions (recombination) occurs by the well-understood collisions processes of Radiative Electron Capture (REC) and NonRadiative Capture (NRC). These processes, which require an electron in the initial state, have cross sections that decrease rapidly with increasing collision energy. REC is the capture of a target electron by the ion with the simultaneous emission of a photon (to balance momentum and energy). NRC is the capture of an electron that is initially bound to a target atom or ion. Until recently, REC and NRC were thought to be the dominant processes for electron capture at all relativistic energies.

The large transient fields produced in relativistic charged particle atomic collisions (no nuclear contact) have long been known to produce electrons through electron-positron pair production [1]. But, in 1984, Gould pointed out that for bare heavy ions, the probability for pair creation with simultaneous capture of the electron from the pair into the K-shell, was

\footnotetext{
${ }^{*}$ Supported by the Director, Office of Energy Research, Office of Basic Energy Sciences, Chemical Sciences Division and (BF) Office of High Energy and Nuclear Physics, Nuclear Physics Division, U.S. Department of Energy under contract DE-AC03-76SF00098. One of us (WEM) was partially supported by NSF grant No. PHY 8614650.
}

significant [2]. The cross section for this "capture from pair production" mechanism is expected to increase with energy (as does the cross section for producing free electron-positron pairs), making it the dominant electron capture mechanism at highly relativistic energies. And since capture from pair production requires no electron in the initial state, it can take place between two bare ions, possibly limiting the lifetime of stored beams of bare heavy ions in relativistic heavy ion colliders.

A number of theoretical papers aimed at calculating the cross sections for electron capture from pair production have been published since 1984 [3]. Different calculational techniques were used, and several results, in disagreement with each other, have been reported. Until recently, no experimental measurement to check the validity of these different theoretical predictions, or even the existence of electron capture from pair production, has existed.

In this article we report the observation and measurement of electron capture from electron-positron pair production in relativistic heavy ion collisions, and discuss the possible implications for the lifetime of heavy ions in the Relativistic Heavy Ion Collider (RHIC) now being constructed at Brookhaven National Laboratory [4]. The experiment has been performed at the Bevalac accelerator at Lawrence Berkeley Laboratory, using $956 \mathrm{MeV} / \mathrm{u}$ bare uranium ions ( ${ }^{92+}$ ) incident on thin, fixed targets of Au.

\section{EXPERIMENT DESCRIPTION}

Figure 1 shows a diagram of the Advanced Positron Spectrometer (APS) used to detect positrons. The $\mathrm{U}^{92+}$ ion passes through a fixed target located inside the APS, described below. In the case of capture from pair production, the electron is created directly bound to the uranium ion, changing its charge by one unit to $\mathrm{U}^{91+}$. The experimental signature is the detection of the positron emitted during the collision, in coincidence with the charge-changed $\mathrm{U}^{91+}$. The $\mathrm{U}^{91+}$ is magnetically separated from the main beam of $\mathrm{U}^{92+}$ and each charge state is detected by a scintillator-photomultiplier tube detector. 


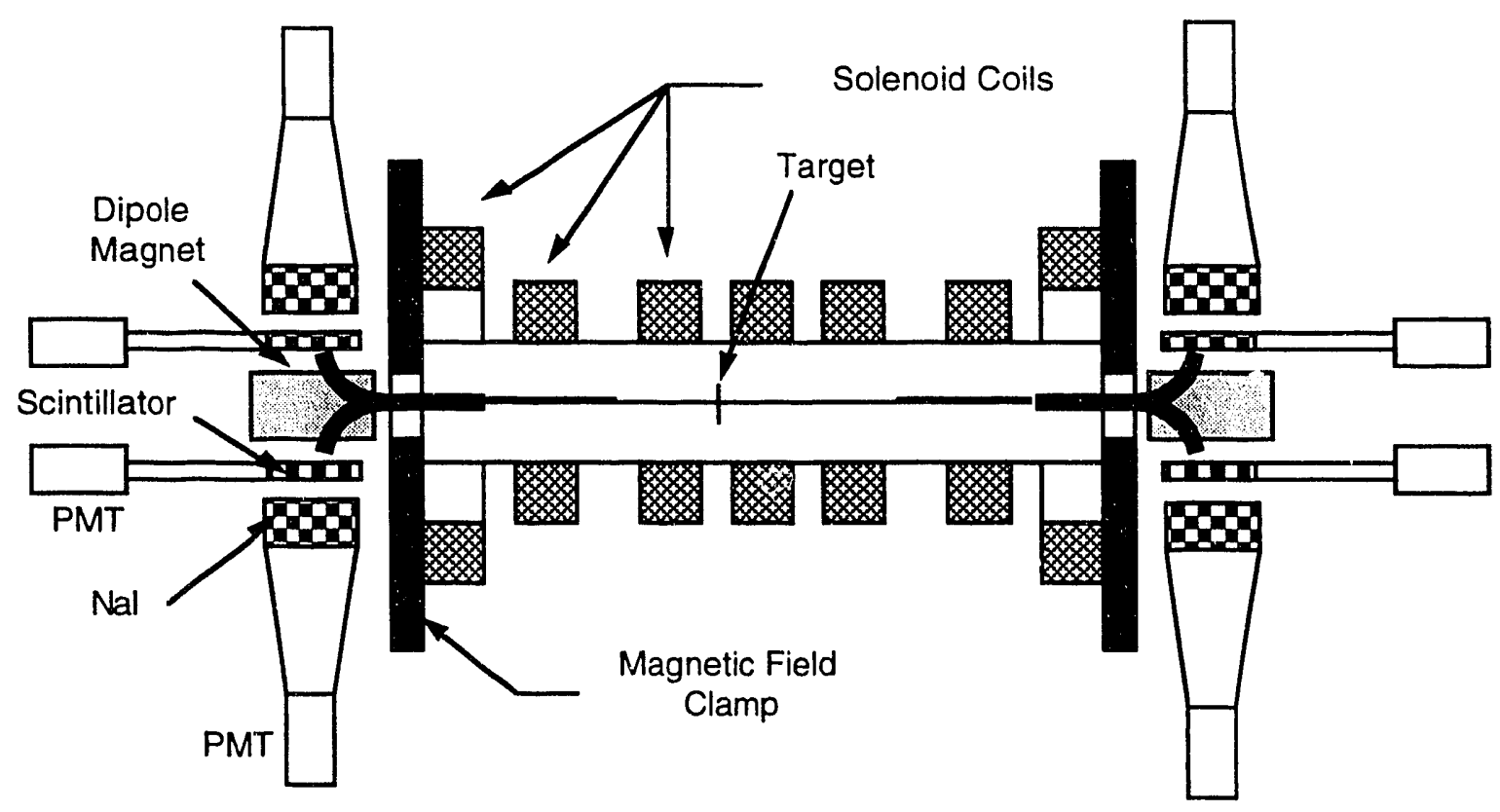

Figure 1 - Schematic diagram of the apparatus (top - sectional view). The solenoid field decreases adiabatically from the center towards the ends causing the particle orbits to grow and their divergence to decrease, allowing them to be swept by the dipole magnets into the detectors. Onl $y$ one positron detector (and no electron detector) is shown. The target is located near the center of the solenoid and the heavy ion beam travels horizontally through the apparatus.

The target is placed inside and slightly upstream from the center of the APS. The APS is used to detect and measure the energy distribution and angular distribution of the electrons and positrons emitted from the target. It contains a solenoid magnet in the center and a dipole magnet at each end. The solenoid generates a strong magnetic field $(B=0.8 \mathrm{~T}$ max.) that adiabatically decreases toward the ends. The field transports the electrons and positrons away from the target, and converts much of the positron (electron) transverse motion into longitudinal motion. The positrons and the electrons are efficiently deflected in opposite transverse directions at each end of the solenoid by the dipoles. There they strike plastic scintillator-photomultiplier tube detectors.

This combination of magnetic fields, results in a very high acceptance for electrons and positrons emitted both forward and backward with respect to the beam direction. Without the adiabatically decreasing field, the acceptance of the spectrometer would be very low because most of the positrons, upon reaching the end of the solenoid field, would have a large transverse motion causing them to strike the walls of the apparatus rather than to be deflected into the spectrometer detectors. Tests of the APS using beams and radioactive sources have shown a detection efficiency close to unity for emission angles of up to 75 degrees forward and backward. The acceptance is independent of the positron or electron energy in the energy range investigated (from 0.1 to $2.5 \mathrm{MeV}$ ).
The emission angle with respect to the beam direction is measured using the time of flight of the positrons (electrons) through the solenoid. Four plastic scintillatordetectors (see figure 1) are used to detect the positrons (electrons) and to measure their energy and time of flight. The magnetically separated $\mathrm{U}^{91+}$ ion that produced the positron, detected in its scintillator-detector at the end of the beamline, is used for the timing reference.

The dipole magnets that deflect electrons and positrons in opposite directions are used for the initial discrimination between electrons and positrons. However, at $1 \mathrm{GeV} / \mathrm{u}$, a large number of knock-on electrons are ejected from the target by collisions with the uranium ion. Approximately 3 to 4 electrons with an energy above $100 \mathrm{keV}$ are ejected from a 1 $\mathrm{mg} / \mathrm{cm}^{2}$ gold target for every uranium ion, while only one positron from the same target is expected for every $10^{6}$ uranium ions. Roughly two or three per thousand knock-on electrons backscatter from the electron scintillator-detector into the positron scintillator-detector, thus simulating a position.

To discriminate against these scattered electrons we require the detection of one of the two $511 \mathrm{keV}$ photons that are emitted back-to-back when the positrons annihilate at rest in the plastic scintillator. The $511 \mathrm{keV}$ photon is detected by a NaI scintillator-photomultiplier detector (see figure 1). The detection efficiency of the photon (by the $12.5 \mathrm{~cm}$ diametar by $15 \mathrm{~cm}$ long active area) NaI detector has been measured to be $42 \%$, with roughly $60 \%$ of the photons appearing as a narrow 
single peak at $511 \mathrm{keV}$, and the rest as a broad Compton distribution. In our data analysis only the narrow peak is used. This sets the overall efficiency of the APS for the detection of a positron at $25 \%$.

\section{RESULTS}

We integrate the spectra over the positron energy and angle to obtain the total cross section for capture from pair production. We correct for the angular region between 75 and 105 degrees, not accounted for by the spectrometer, and for the energy range above $2.5 \mathrm{MeV}$. Making these small corrections, we measure the total cross section for capture from pair production by a $956 \mathrm{MeV} / \mathrm{u} \mathrm{U}^{92+}$ on a gold target to be $2.19(0.25)$ barns.

We obtain a cross section of $3.3(0.65)$ for the free pair process by assuming that a similar fraction of free pairs are not detected by the spectrometer. (The error we make, in estimating the fraction between 75 and 105 degrees for free pair production is well within our error bars, even though the angular distributions for capture from pair production and free pair production are expected to be different.)

\section{DISCUSSION}

At $1 \mathrm{GeV} / \mathrm{u}$ the elcctron of the $\mathrm{e}^{+}-\mathrm{e}^{-}$pair is almost as likely to emerge from the collision bound to the $\mathrm{U}^{92+}$, changing its charge state to $\mathrm{U}^{91+}$, as it is to emerge free. Perturbation theory predictions [5] underestimate the measured total cross section for capture from pair production by about a factor of 2.5, and underestimate the ratio of capture to free pair production by about a factor of 3 [6].

Calculations of the cross section for both free pair production and capture from pair production have been made for Au at RHIC collider energies of $100+100 \mathrm{GeV} / \mathrm{u}$. These perturbative calculations indicate that the free pair production cross section increases to the order of $3 \times 10^{5}$ barns for $\mathrm{Au}$ ions, while the fraction of pair electrons that are captured is predicted to decline to a part per thousand at RHIC energies $[4,7]$. The fraction of electrons that are captured may be sensitive, however, to several effects such as multiple pair production, combined charges of projectile and target, and other effects enhancing capture. As has been seen, the fraction at $1 \mathrm{GeV} / \mathrm{u}$ is observed to be considerably larger than that predicted by perturbation theory.
Capture from pair production (resulting in the loss of the charge-changed ion in the collider ring) has been predicted to be the dominant beam loss mechanism foi colliding $\mathrm{Au}+$ $\mathrm{Au}$ beams at RHIC and a significant loss mechanism for lighter ions [4]. Small effects that might enhance capture, as discussed above, could have a significant impact upon the ratio of captured electrons, and thus upon the operation of RHIC. If the fraction of captured electrons is an order of magnitude higher than predicted at RHIC energies, then the current after 10 hours will decrease to about $20 \%$ of the initial value, instead of $77 \%$, after taking into account intrabeam Coulomb scattering.

Measurements of the cross section for capture from pair production at Bevalac energies indicate that current calculations based upon perturbation theory underestimate the fraction of electrons captured by a factor of three. These results indicate the need for better calculations and measurements over a wide range of energies to understand the useful lifetime of heavy ions in RHIC.

\section{REFERENCES}

[1] G. Racah, Nuovo Cimento 14, 93 (1937) and references therein.

[2] H. Gould, Lawrence Berkeley Laboratory Report No. LBL-18593 (1984).

[3] See references in A. Belkacem, Harvey Gould, B. Feinberg, R. Bossingham, and W.E. Meyerhof, "Measurement of Electron Capture From $\mathrm{e}^{+}-\mathrm{e}^{-}$Pair Production in Relativistic Heavy Ion Collisions," submitted to Phys. Rev. Lett. (1993).

[4] "Conceptual Design of the Relativistic Heavy Ion Collider - RHIC", BNL Report BNL-52195 pp 117-121.

[5] U. Becker, J. Phys. B20, 6563 (1987); values for U on Au were computed by $\mathrm{K}$. Momberger.

[6] U. Becker, N. Grün and W. Scheid, J. Phys. B19, 1347 (1986).

[7] C. Bottcher, M.R. Strayer, Phys. Rev. D39, 1330 (1989). 

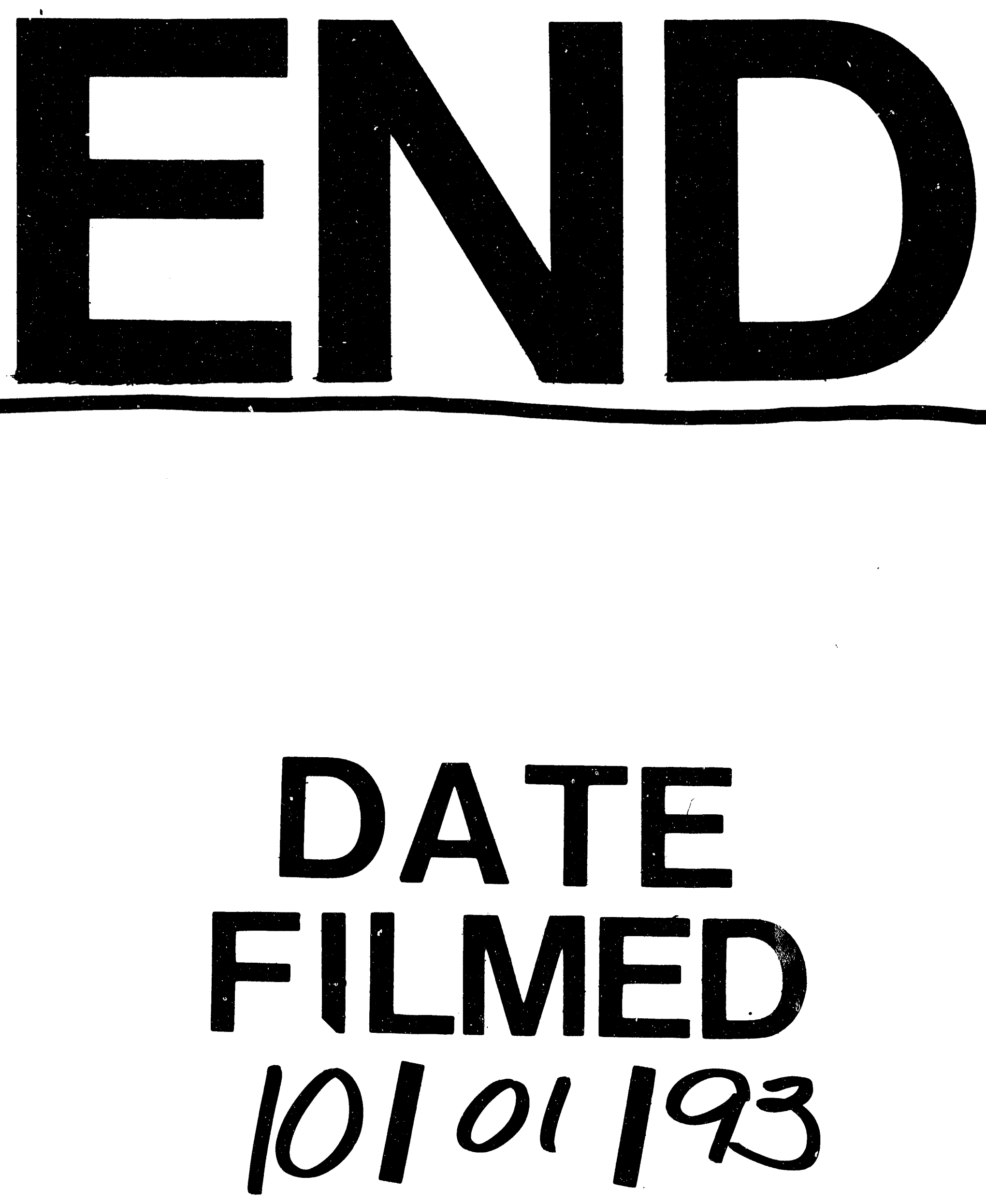

1 
